\title{
Structural Development in Cycloolefin Copolymers Under Uniaxial Elongational Flow
}

\author{
Pralay Maiti, ${ }^{1}$ Masami Okamoto ${ }^{2}$ \\ ${ }^{1}$ Material Science and Engineering, Cornell University, Bard Hall, Ithaca, New York 14853-1501 \\ ${ }^{2}$ Advanced Polymeric Materials and Engineering, Toyota Technological Institute, Hisakata 2-12-1, Tempaku, Nagoya \\ 468-8511, Japan
}

Received 28 December 2002; accepted 16 July 2003

\begin{abstract}
Development of structures in new cycloolefin copolymers (COCs) of ethylene-norbornene (E-NB) and ethylene-tetracyclododecene (E-TD) of different NB/TD compositions were investigated under uniaxial elongational flow. For E-NB copolymer, which has multiblock sequences, a shoulder in WAXD at $0.76 \mathrm{~nm}$ was evident besides its amorphous halo, and the relative intensity of the peak increased with increasing comonomer content (NB). The appearance of a new peak for E-TD copolymer, and a relatively higher intense peak for E-NB copolymer at the same position of $0.76 \mathrm{~nm}$, indicated that local ordering of the segments occurred when the COC was subjected to uniaxial flow. A lower comonomer composition of elongated COC, at
\end{abstract}

a constant Hencky strain rate, exhibited two $T_{g}$ values, whereas a higher composition showed a single $T_{g}$. Both local ordering and segmentization occurred in low comonomer content COCs but only local ordering occurred in higher comonomer content COCs. Both the shear viscosity and roll processing experiments exhibited similar phenomena of segregation. A model is proposed to illustrate the structure of COC after uniaxial elongation/deformation. (c) 2004 Wiley Periodicals, Inc. J Appl Polym Sci 91: 3421-3427, 2004

Key words: elongational flow; cycloolefin copolymers; glass transition; rheology; uniaxial

\section{INTRODUCTION}

A new class of photonics material, emerging from metallocene technology, is that of cycloolefin copolymers (COCs), which are the copolymers of norbornene (NB) or tetracyclododecene (TD) with ethylene. The composition and the stereoselectivity of the comonomers (NB/TD) can be controlled by using different catalysts. In general COCs are amorphous in nature, as evidenced by the absence of any distinct melting and the absence of a scattering pattern in Rayleigh light scattering. The glass-transition temperature can be varied from 0 to $250.0^{\circ} \mathrm{C}$ by changing the NB/TD content in the copolymer. Polymers of this kind have strong potential to replace conventional thermoplastics like polycarbonate and poly(methyl methacrylate). High chemical resistance and low birefringence are among the improved properties of COCs compared to those of conventional polyolefins. The elongational rheology ${ }^{1-3}$ was investigated by our newly developed elongational flow optorheometry, which enabled us to measure simultaneously the transient tensile stress $\sigma\left(\dot{\varepsilon}_{0} ; t\right)$ and birefringence $\Delta n\left(\dot{\varepsilon}_{0} ; t\right)$ as a function of time under uniaxial elongation with constant Hencky strain rate $\dot{\varepsilon}_{0}$. So far, however, there has been no report about

Correspondence to: P. Maiti (pm82@cornell.edu).

Journal of Applied Polymer Science, Vol. 91, 3421-3427 (2004) (C) 2004 Wiley Periodicals, Inc. the structural development in COCs under uniaxial elongation in the melt state. Ex situ measurement in wide-angle X-ray diffraction (WAXD), differential scanning calorimetry (DSC), roll processing, shear viscosity, and ${ }^{13} \mathrm{C}-\mathrm{NMR}$ were conducted on COCs recovered before and after elongation. The interesting features are reported here with a plausible mechanism of the development of structure.

\section{EXPERIMENTAL}

\section{Materials}

Ethylene-norbornene (E-NB) and ethylene-tetracyclododecene (E-TD) copolymers ${ }^{4}$ (Scheme 1) of different NB/TD content were prepared with metallocene and Ziegler-Natta catalyst, respectively. ${ }^{13} \mathrm{C}-\mathrm{NMR}$ spectra, taken by the supplier, show that E-NB copolymers are of the multiblock type, having small block sequences, whereas E-TD copolymers are all random copolymers. The molecular weights were measured by using a high-temperature gel permeation chromatograph $^{3}$ (GPC: Tosoh HLC-8121 GPC/HT) with polystyrene standard and o-dichlorobenzene as carrier. The characteristics of the copolymers are summarized in Table I.

\section{WAXD studies}

The X-ray diffraction experiments were performed using an MXlabo diffractometer (MAC Science Co.) with 


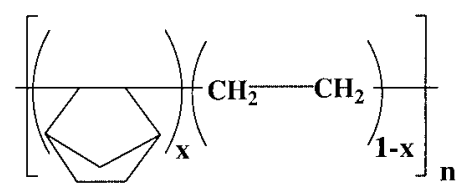

Ethylene-norbornene (E-NB) copolymer

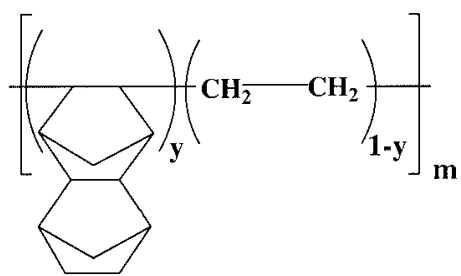

Ethylene-tetracyclododecene (E-TD) copolymer

Scheme 1

$\mathrm{Cu}-\mathrm{K}_{\alpha}$ radiation and graphite monochromator at room temperature with the samples before and after elongation. The scan rate was $0.5^{\circ} / \mathrm{min}$ for each experiment.

\section{Differential scanning calorimetry}

Glass-transition temperatures, before and after elongation, were measured by use of a differential scanning calorimeter (TA2920; TA Instruments, New Castle, DE) at the heating rate of $5.0^{\circ} \mathrm{C} / \mathrm{min}$. The DSC was calibrated with indium before use.

\section{Elongational flow rheometry (EFR)}

In EFR, using a commercialized Meissner's new elongational rheometer, a specimen of about $60 \times 7 \times 0.5$

TABLE I

Characteristics of E-NB and E-TD Copolymers

\begin{tabular}{cccc}
\hline $\begin{array}{c}\text { Ingredient } \\
(\text { mol \%) }\end{array}$ & $T_{g} /\left({ }^{\circ} \mathrm{C}\right)$ & $M_{w} \times 10^{-4}$ & $M_{w} / \mathrm{M}_{n}$ \\
\hline $\mathrm{NB}$ & & & \\
$28^{\mathrm{a}}$ & 76.0 & 12.0 & 2.1 \\
$50^{\mathrm{a}}$ & 150.0 & 9.0 & 2.4 \\
52 & 132.0 & 9.9 & 2.2 \\
$60^{\mathrm{a}}$ & 172.0 & 11.1 & 2.8 \\
$\mathrm{TD}$ & & & \\
20 & 64.0 & 12.5 & 2.7 \\
30 & 103.0 & 10.0 & 3.1 \\
41 & 146.0 & 10.4 & 2.4 \\
48 & 169.0 & 12.5 & 2.7 \\
\hline
\end{tabular}

${ }^{\text {a }}$ Multiblock type with short block length.

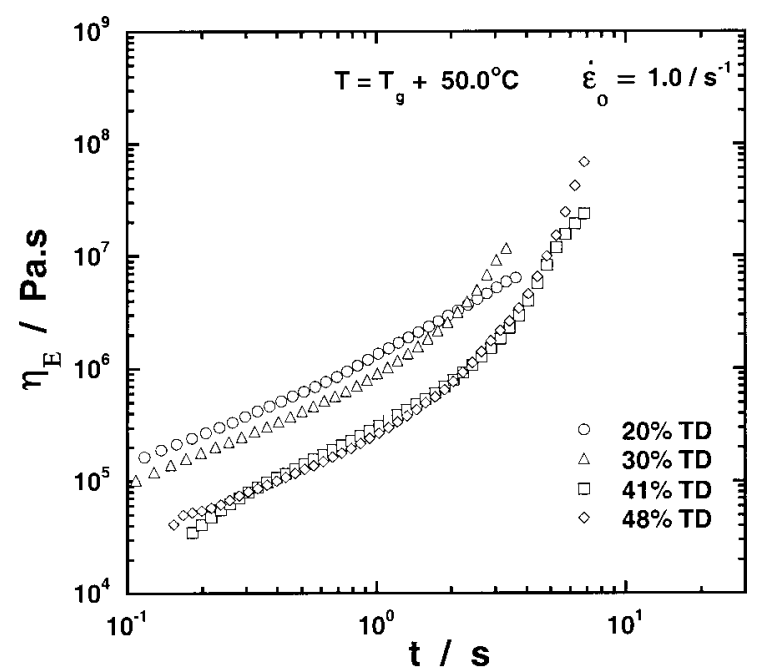

Figure 1 Double-logarithmic plots of transient elongational viscosity $\eta_{E}\left(\dot{\varepsilon}_{0} ; t\right)$ as a function of time for different TD contents indicated at $T_{g}+50^{\circ} \mathrm{C}$ with a constant Hencky strain rate $\left(\dot{\varepsilon}_{0}=1.0 \mathrm{~s}^{-1}\right)$.

$\mathrm{mm}^{3}$ in size was annealed above $T_{g}$ for $3 \mathrm{~min}$ and subjected to uniaxial elongation with a constant Hencky strain rate $\dot{\varepsilon}_{0}$ in the range of 1.0 to $0.01 \mathrm{~s}^{-1}$. The details of the instrumentation are described elsewhere. ${ }^{5}$ The elongated samples were quenched to room temperature in between two steel plates and $e x$ situ measurements were done in WAXD and DSC.

\section{Steady-shear viscosity}

Steady-shear viscosity in the liquid state was measured, using steady-shear tests, on a Rheometrics dynamic analyzer (RDAII; Rheometrics, Amherst, MA) using cone-plate geometry $(25 \mathrm{~mm})$ at various temperatures between $T_{g}+80$ to $T_{g}+160^{\circ} \mathrm{C}$ at various shear rates $\left(\dot{\gamma}=0.01-1.0 \mathrm{~s}^{-1}\right)$. Stress relaxation experiments in step-shear deformation were conducted with parallel-plate geometry $(25 \mathrm{~mm})$.

\section{Roll processing}

The samples were roll processed (Oriental Motor Co.; M590-501C) at different temperatures higher than glass-transition temperature. The diameter, rotating speed, and clearance of roll were $76 \mathrm{~mm}, 22 \mathrm{rpm}$, and $0.5 \mathrm{~mm}$, respectively.

\section{RESULTS AND DISCUSSION}

\section{Elongational viscosity}

Figure 1 shows the extensional viscosity of E-TD copolymers with time at $T_{g}+50^{\circ} \mathrm{C}$ with the Hencky strain rate $\left(\dot{\varepsilon}_{0}\right)$ of $1.0 \mathrm{~s}^{-1}$. Results obey the Trauton's rule. The increasing tendency of viscosity, often called 


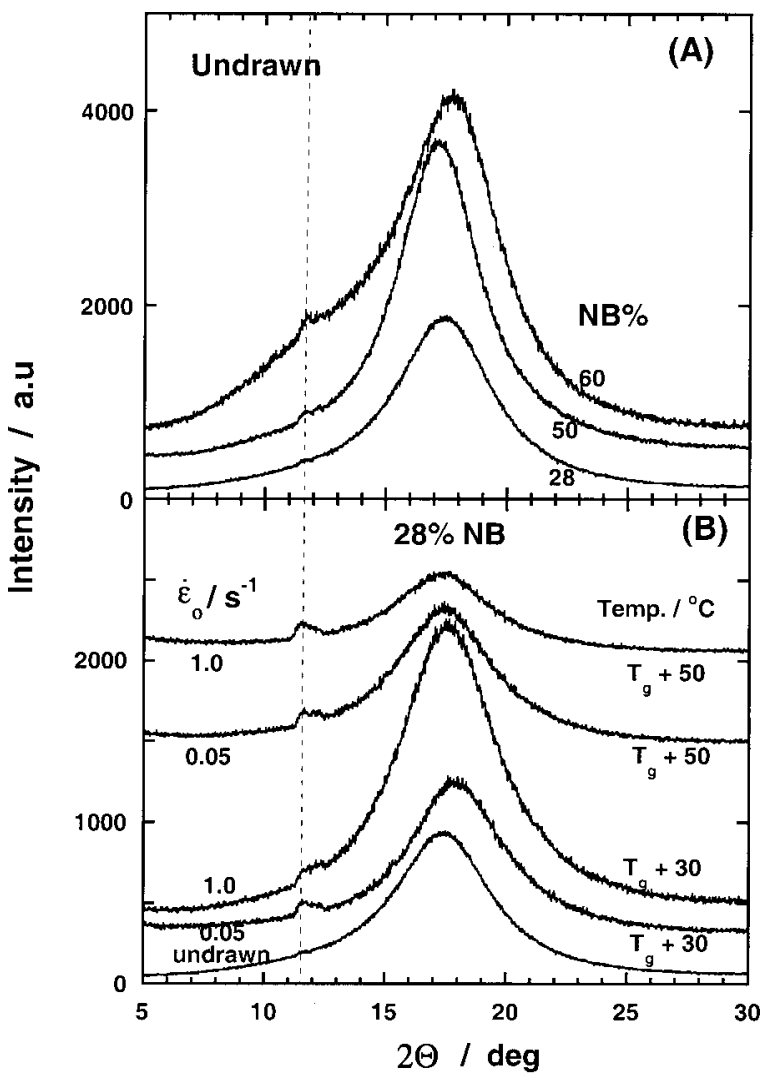

Figure 2 WAXD patterns of E-NB copolymers of different NB contents: (A) undrawn samples of indicated NB content; (B) comparison between undrawn and elongated samples at indicated temperature; and Hencky strain rates $\dot{\varepsilon}_{0}$. The dotted line indicates the position side peak.

strain-induced hardening, increases with TD content. The E-NB copolymers exhibit the same behavior at different temperatures of measurement. Initial viscosity gradually decreases with increasing comonomer content as the measurement temperature $\left(T_{g}+50^{\circ} \mathrm{C}\right)$ increases with comonomer content, which decreases the viscosity of the system. The details of the elongational viscosity test were discussed in our earlier publications. ${ }^{2,3}$

\section{Structural development}

WAXD patterns of undrawn E-NB copolymers, shown in Figure 2(A) exhibit a liquid-like halo (correlation length of $0.51 \mathrm{~nm}$ ) along with a small side peak (shown by the dotted line) of correlation length 0.75 $\mathrm{nm}$, the intensity of which increases with comonomer content. This result is in agreement with the work of Haselwander et al. ${ }^{6}$ and Rische et al., ${ }^{7}$ who showed that the side peak becomes prominent with increasing molecular weight of NB homopolymer and the comonomer content of E-NB copolymer, respectively. Figure 2(B) compares the WAXD patterns (before and after elongation) of $28 \mathrm{~mol} \% \mathrm{NB}$ content E-NB copol- ymer using various stretching conditions ( $T$ and $\dot{\varepsilon}_{0}$ ), and shows the prominent side peak (correlation length $=0.75 \mathrm{~nm}$ ) after elongation compared to the unelongated one. On the other hand, unelongated E-TD copolymers always show only one peak (liquid-halo) irrespective of comonomer composition, even for high comonomer content E-TD (Fig. 3). After elongation, however, E-TD copolymers also exhibit a side peak corresponding to the correlation length of $0.76 \mathrm{~nm}$ in every stretching condition ( $T$ and $\dot{\varepsilon}_{0}$ ). The absence of a scattering pattern in light scattering and the absence of an endothermic peak in the DSC thermogram when heated up to $300^{\circ} \mathrm{C}$ indicate that COCs (any comonomer content) do not crystallize even after uniaxial elongation. In summary, after uniaxial elongation of both the E-NB and E-TD copolymers, some local ordering takes place.

\section{Glass-transition temperature of developed structure}

When total heat flow is measured against temperature, lower comonomer content $(28 \mathrm{~mol} \% \mathrm{NB}) \mathrm{E}-\mathrm{NB}$ copolymer shows two $T_{g}$ values [Fig. 4(A)] after elongation, whereas it exhibited only one $T_{g}$ before stretching, behaving like a normal copolymer. The dotted line represents the $T_{g}$ before stretching and the arrows show the $T_{g}$ of the elongated specimens. On the other hand, higher comonomer content E-NB copolymers (>30 mol \%) always show a single $T_{g}$, even after elongation, when stretched at different temperatures and strain rates [Fig. 4(B)]. With respect to composi-

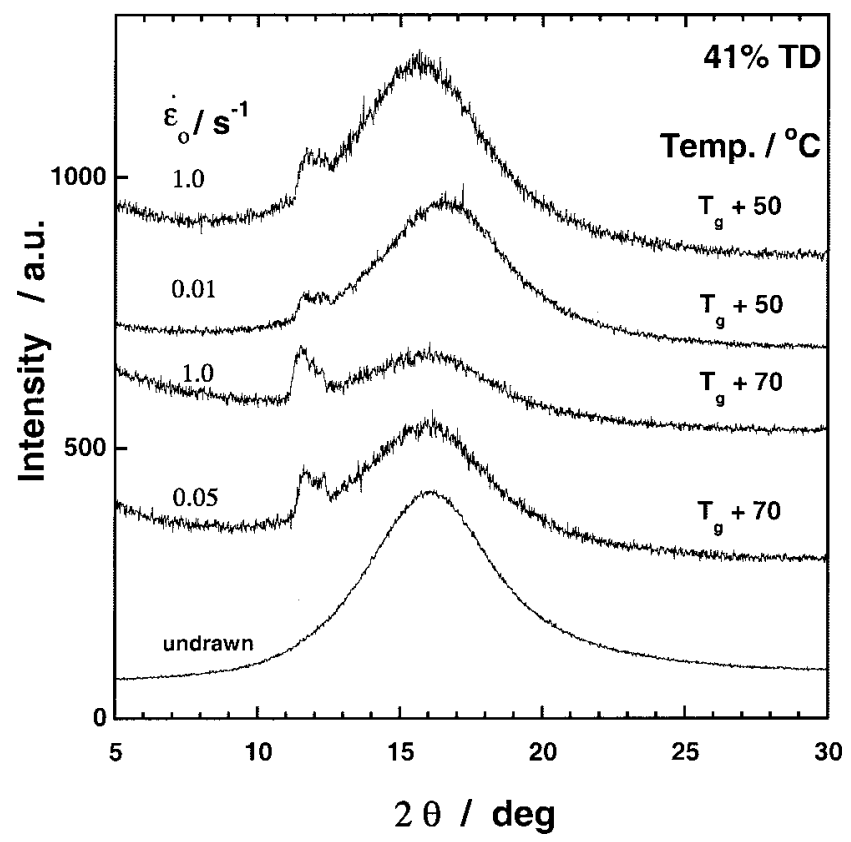

Figure 3 WAXD patterns of $41 \mathrm{~mol} \%$ TD content E-TD copolymer of undrawn sample elongated at the indicated conditions of temperature and strain rates $\dot{\varepsilon}_{0}$. 

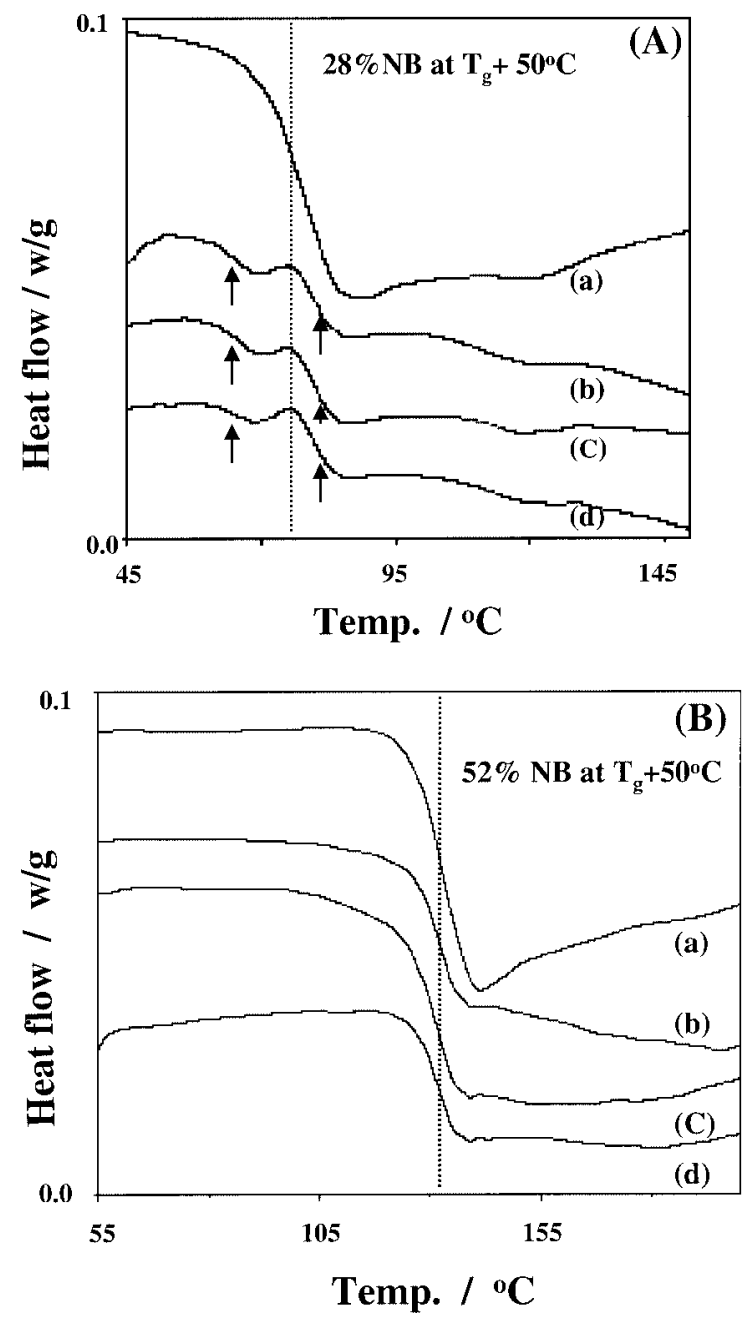

Figure 4 DSC thermograms of E-NB copolymers before and after elongation. (A) $28 \mathrm{~mol} \% \mathrm{NB}$ content E-NB copolymer elongated at $T_{g}+50.0^{\circ} \mathrm{C}$ : (a) undrawn sample at room temperature, (b) $\dot{\varepsilon}_{0} \stackrel{g}{=} 0.75 \mathrm{~s}^{-1}$, (c) $\dot{\varepsilon}_{0}=0.3 \mathrm{~s}^{-1}$, (d) $\dot{\varepsilon}_{0}=0.05$ $\mathrm{s}^{-1}$. The dotted line represents the $T_{g}$ of the $28 \mathrm{~mol} \% \mathrm{E}-\mathrm{NB}$ copolymer in the undrawn state. The arrows indicate the $T_{g}$ after elongation. (B) $52 \mathrm{~mol} \% \mathrm{NB}$ content E-NB copolymer at $T_{g}+50.0^{\circ} \mathrm{C}$ : (a) undrawn sample, (b) $\dot{\varepsilon}_{0}=0.75 \mathrm{~s}^{-1}$, (c) $\dot{\varepsilon}_{0}$ $=0.3 \mathrm{~s}^{-1}$, (d) $\dot{\varepsilon}_{0}=0.1 \mathrm{~s}^{-1}$. The dotted line represents $T_{g}$ of the $52 \mathrm{~mol} \% \mathrm{E}-\mathrm{NB}$ copolymer in the undrawn state.

tion, E-TD copolymers display exactly the same behavior as that of E-NB copolymers; that is, low TD content copolymers ( $\leq 30 \mathrm{~mol} \%)$ show two $T_{g}$ values and high TD content ( $>30 \mathrm{~mol} \%$ ) copolymers exhibit a single $T_{g}$ after uniaxial elongation.

The enhanced strain-induced hardening of higher $\mathrm{NB} / \mathrm{TD}$ comonomer content copolymer indicates the anchoring effect of NB/TD moieties in the main chain, other than the entanglement, which is supposed to be less for higher NB/TD content copolymer. Because the molecular weights are almost the same for low and high comonomer content copolymers, the degree of polymerization $D P_{w}$ gradually decreases with increasing comonomer content. ${ }^{3}$ There is no evidence of crosslinking at high temperatures because the molecular weights, measured using GPC, are the same before and after elongation for the whole composition range studied here. The side peak in WAXD of unelongated E-NB copolymer, especially at higher comonomer content $(>30 \mathrm{~mol} \%)$, arises from the blocky nature of E-NB copolymers (which have small block sequences), which is categorically absent in random E-TD copolymer, but when elongated it also shows a small peak (correlation length $0.75 \mathrm{~nm}$ ) and two $T_{g}$ values. To summarize, the results clearly show that some local ordering occurs after elongation for the whole composition range of E-NB and E-TD copolymers and the DSC results (two $T_{g}$ values) also indicate the formation of soft and hard segments for the low $\mathrm{NB} / \mathrm{TD}$ content $(<30 \mathrm{~mol} \%)$ copolymer after uniaxial elongation. The single $T_{g}$ and prominent side peak of high comonomer content COCs strongly suggest that local ordering occurs after elongation but segmentization does not occur because of the much higher extent of NB/TD comonomer in the main chain. Here, it can be mentioned that the possibility of conformational change has been ruled out because the solid-state ${ }^{13} \mathrm{C}$ NMR showed exactly the same patterns before and after elongation for the whole composition range.

On the basis of the experimental observations, a molecular mechanism has been proposed for the uniaxial elongation of COCs (Scheme 2). During uniaxial elongation, the polymer chains are stretched and, as a result, the anchoring effect of comonomer becomes prominent, giving rise to higher extensional viscosity for higher NB/TD content copolymer. After elongation, the stretched molecules are aligned, and for low comonomer content copolymer, the formation of softand hard-segment zones is obvious, depending on the conformation and/or position of the comonomer, and the NB/TD anchored part would be the hard segment having high $T_{g}$ with the remainder being the soft segment (low $T_{g}$ ) (Scheme 2). For higher comonomer content copolymer, because of the densely populated NB/TD comonomer, separate segments cannot be formed after elongation and thus remain uniform throughout the molecular region, behaving like normal copolymer and exhibiting one $T_{g}$. The side peak in WAXD appears from the correlation of the anchored portion, which is supposed to have higher correlation length than the normal $\mathrm{C}-\mathrm{C}$ correlation length of a liquid-like structure.

\section{Roll processing}

The alignment of COCs upon uniaxial extension should have some effect on the viscosity of the system and thus we roll-processed the low and high comonomer content COCs and found that viscosity of the low NB/TD content copolymer decreased significantly after roll processing, whereas shear viscosity of the high 


\section{Molecular mechanism of chain extension of COC under uniaxial flow : Schematic representation}

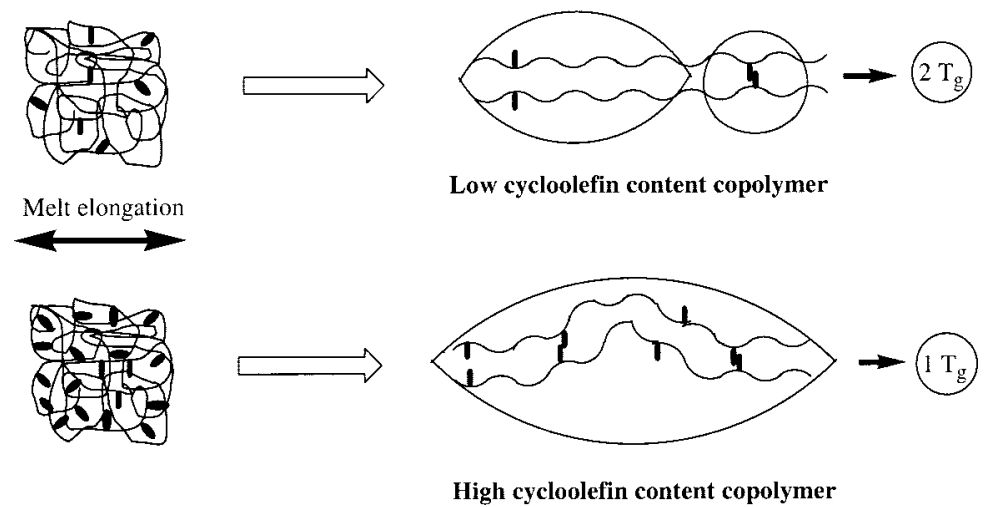

Scheme 2 Schematic representation of molecular mechanism of chain extension of COC under uniaxial flow.

NB/TD content copolymers decreased very slightly after roll processing (Fig. 5), because the much higher amount of bulkier NB/TD groups in the main chain made alignment and disentanglement of the contents difficult. Like uniaxial elongation, lower comonomer content copolymer exhibits two $T_{g}$ values after roll processing but higher comonomer content copolymers always show a single $T_{g}$. In brief, phenomenologically,

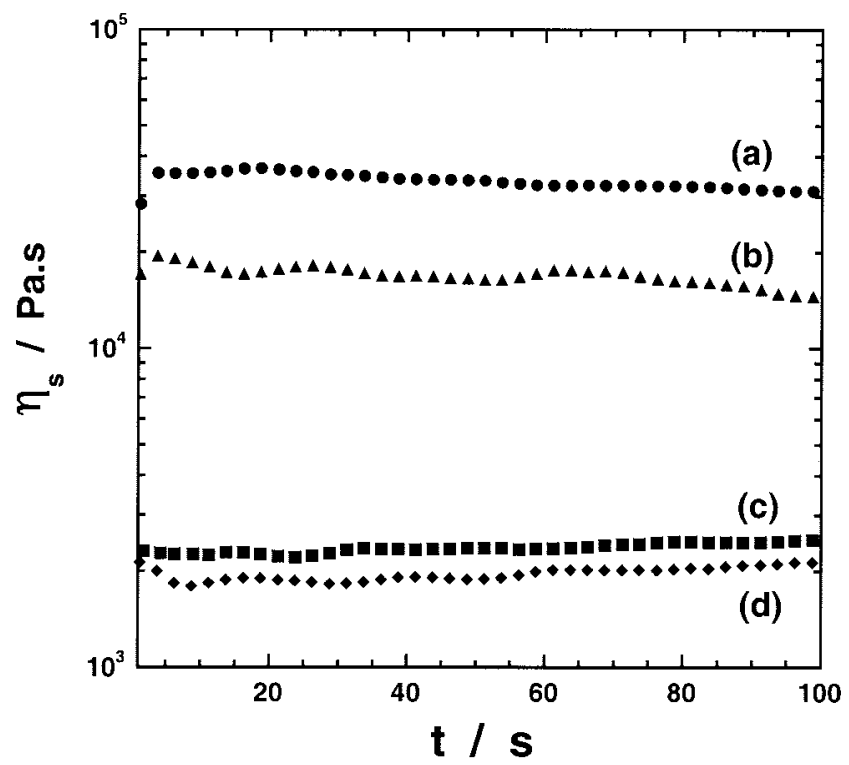

Figure 5 Steady-shear viscosity of the sheared samples against time at $\dot{\gamma}=1.0 \mathrm{~s}^{-1}$ : (a) $20 \mathrm{~mol} \%$ TD copolymer before roll processing, measured at $160.0^{\circ} \mathrm{C}$; (b) $20 \mathrm{~mol} \% \mathrm{TD}$ roll processed at $160.0^{\circ} \mathrm{C}$ for 80 times, measured at $160.0^{\circ} \mathrm{C}$; (c) $41 \mathrm{~mol} \% \mathrm{TD}$ copolymer before roll processing, measured at $240.0^{\circ} \mathrm{C}$; (d) $41 \mathrm{~mol} \%$ TD copolymer roll processed at $240.0^{\circ} \mathrm{C}$, measured at $240.0^{\circ} \mathrm{C}$. roll processing also supports the segmentization of the lower comonomer content COCs.

\section{Shear viscosity}

\section{Temperature dependency}

Temperature dependencies of shear viscosity $\eta_{s}$ of both low and high comonomer content copolymers are shown in Figure 6(A) and (B) at $\dot{\gamma}=1.0 \mathrm{~s}^{-1}$. For low TD content copolymer, sheared in a lower-temperature region, after a certain shear $\eta_{s}$ gradually decreases with time and the extent of this decrease becomes increasingly less with the increase of temperature [Fig. 6(A)]. The onset of decreasing $\eta_{s}$ is shifted toward higher time with increasing temperature. Finally at $T \geq 220^{\circ} \mathrm{C}$ the viscosity becomes constant with time. Like uniaxial elongation, heat flow behavior shows two $T_{g}$ values after shearing at lower temperature, whereas a single $T_{g}$ is observed after shearing at $T \geq 220^{\circ} \mathrm{C}$. On the contrary, $\eta_{s}$ is always constant with time measured at different temperature ranges for higher TD content E-TD copolymer [Fig. 6(B)] and always exhibits a single $T_{g}$ after being sheared at any temperature. The decrease of viscosity with temperature is as usual with other polymeric liquids but the deviation from linearity is believed to be attributable to orientation of molecules after a certain critical shear. At high temperature, viscosity is so low that orientation cannot occur because of the short relaxation time under shear force field. Similarly, the higher comonomer content copolymer cannot disentangle properly because of heavily crowded bulky comonomer, and as a result orientation cannot occur during shear. Degradation of polymer restricts the measurement of shear viscosity beyond $240.0^{\circ} \mathrm{C}$ for $41 \%$ TD content copolymer. This shear experiment is additional support for segmentization occurring in lower monomer content COCs while being 

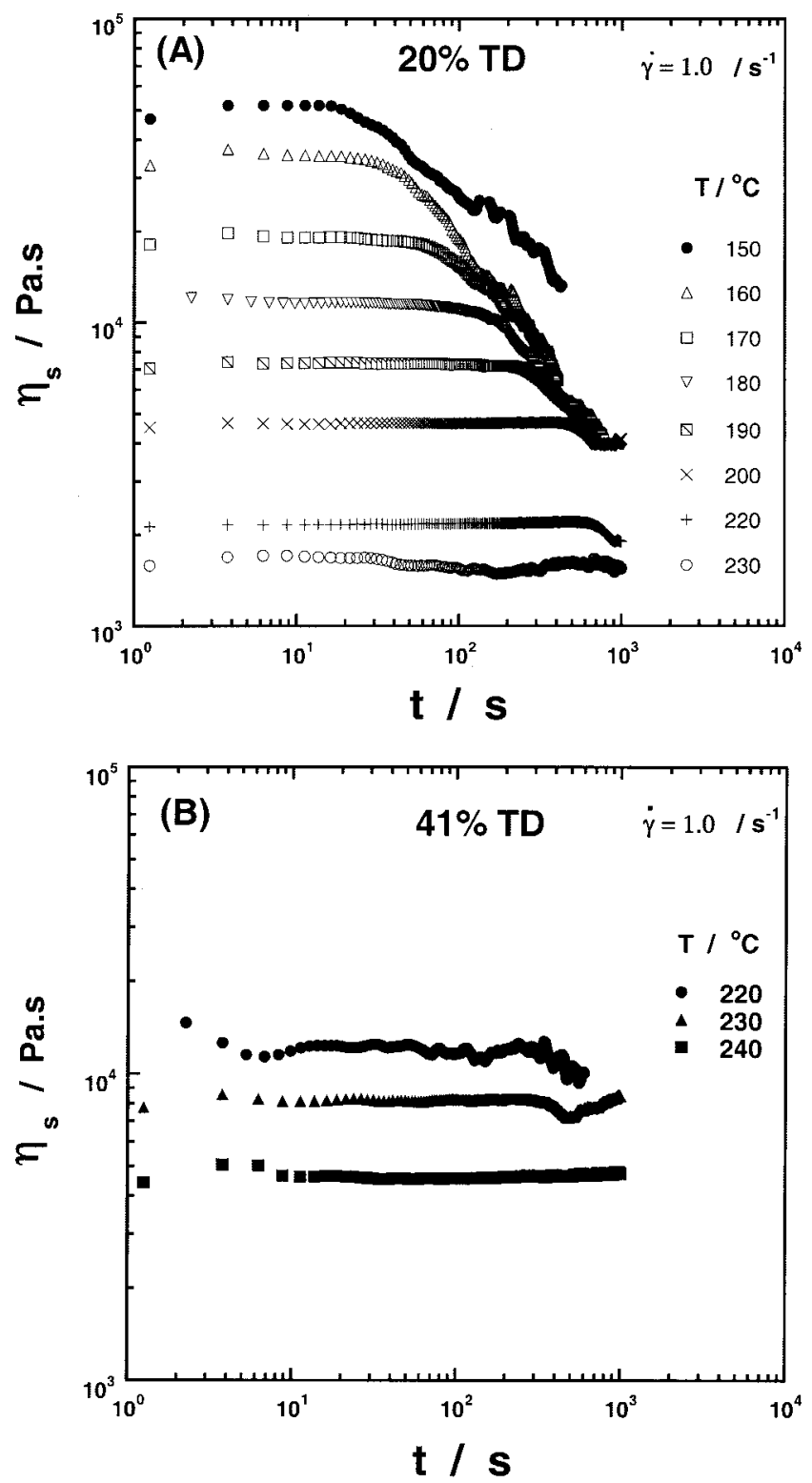

Figure 6 Steady-shear viscosity versus time at various temperatures of measurement showing in plot at $\dot{\gamma}=1.0 \mathrm{~s}^{-1}$ : (A) $20 \mathrm{~mol} \%$ E-TD copolymer, (B) $41 \mathrm{~mol} \%$ E-TD copolymer.

sheared at low temperatures. In the literature, there are reports about structural change, specifically molecular orientation, under shear flow. ${ }^{8-12}$ Ugaz et al. ${ }^{10}$ demonstrated quantitatively the molecular orientation on the shear rate and it increases slightly with undercooling for thermotropic copolyester having a rigid mesogen group. Similarly, following their conclusion, this explains well the segmentization of COCs upon deformation such as shear or uniaxial elongational flow.

\section{Shear rate dependency}

Figure 7(A) and (B) show the shear rate dependency of viscosity for lower and higher comonomer content copolymer, respectively. At low shear rate, $\eta_{s}$ is constant throughout the measurement time studied here. At higher shear rate, however, $\eta_{s}$ starts to decrease and the onset of which shifts toward lower time [Fig. 7(A)]. It must be mentioned that there is no flowout of samples from the disc while shearing even at the high shear rate mentioned here. A 20 wt \% TD content E-TD shows two $T_{g}$ values after shearing at $\dot{\gamma}=0.4$ $\mathrm{s}^{-1}$ (or higher rate), whereas $20 \mathrm{wt} \%$ TD sheared at lower rate (or higher comonomer content copolymer sheared at any rate), where $\eta_{s}$ is always constant irrespective of shear rate [Fig. 7(B)], exhibits a single $T_{g}$. This behavior is exactly similar to the uniaxial elongational behavior of COCs.
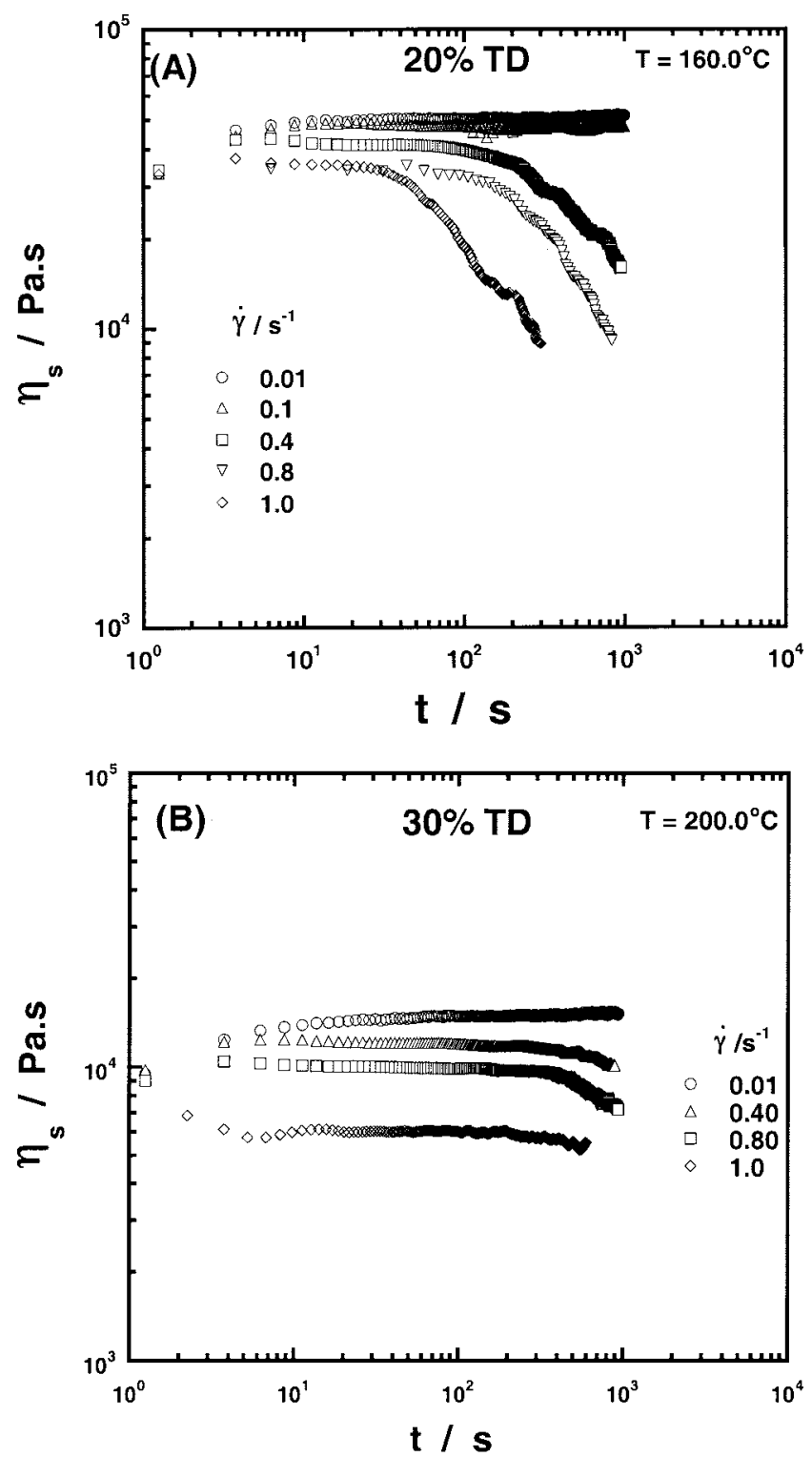

Figure 7 Steady-shear viscosity against time at various shear rates indicated in the figure: (A) $20 \mathrm{~mol} \% \mathrm{E}-\mathrm{TD}$ copolymer measured at $160.0^{\circ} \mathrm{C}$; (B) $30 \mathrm{~mol} \% \mathrm{E}-\mathrm{TD}$ copolymer measured at $200.0^{\circ} \mathrm{C}$. 


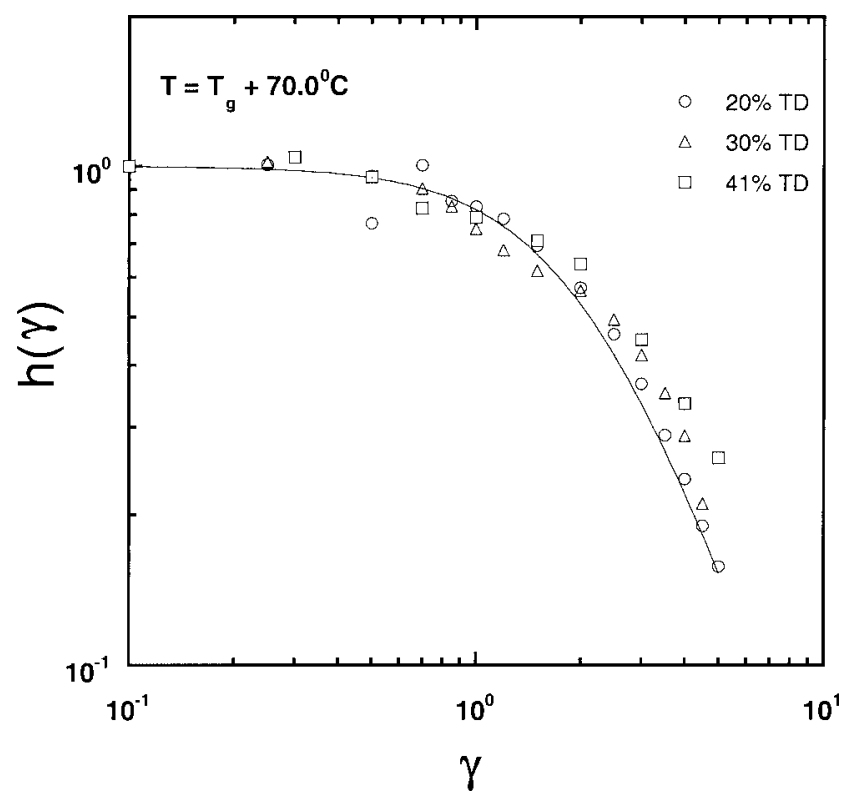

Figure 8 Damping function of various TD content E-TD copolymers measured at temperature $T=T_{g}+70.0^{\circ} \mathrm{C}$ of each copolymer. The solid curve represents the prediction from Doi-Edwards theory.

In brief, from uniaxial elongational flow and shear experiments, we observed that segmentization occurs in lower NB/TD content COCs, after becoming successfully aligned in the direction of flow. In our earlier report ${ }^{3}$ we showed the validity of the "stress optical rule" (SOR) for lower comonomer content, wherease SOR is invalid for high comonomer content COCs. However, strain-induced hardening behavior is prominent without segmentization for higher comonomer content COCs, which is mainly believed to be attributed to the rigid structure. To check the rigidity of the molten COCs of different comonomer contents, a stress-relaxation experiment in step-shear deformation was performed in parallel disc geometry and corrections were made as proposed by Soskey and Witer. ${ }^{13}$

$$
G(t, \gamma)=G_{a}(t, \gamma)\left(1+\partial \ln G_{a}(t, \gamma) / 4 \partial \ln \gamma\right)
$$

Stress relaxation at $\gamma=0.1$ was regarded as the linear relaxation modulus $G^{\circ}(t)$. As with the other polymeric liquids, the shear relaxation modulus $G(t, \gamma)$ can be factored into a linear relaxation modulus $G^{\circ}(t)$ and strain-dependency function, often called damping function $h(\gamma)$, in the long-time region, which is also supported by Doi-Edwards theory. ${ }^{14}$

$$
G(t, \gamma)=G^{\circ}(t) h(\gamma)
$$

The damping function is plotted as a function of strain $\gamma$ for different comonomer contents in Figure 8. The solid line represents the Doi-Edwards equation and it is obvious that, with increasing comonomer content, the deviation (strain hardening) increases. Again, $h(\gamma)$ can be fitted with a strain-dependent function as ${ }^{15,16}$

$$
h(\gamma)=1 /\left(1+a \gamma^{2}\right)
$$

The parameter $a$ is about 0.222 according to Doi-Edwards theory. The best-fit parameters, according to eq. (3), are $0.192,0.165$, and 0.147 for 20,30 , and $41 \mathrm{wt} \%$ TD content E-TD copolymers, respectively. This confirms that higher comonomer content COC makes the damping function more strain hardening and, in other words, the rigidity increases with comonomer content of COCs.

\section{CONCLUSIONS}

In conclusion, during uniaxial elongation, the anchoring effect becomes prominent for higher comonomer content COCs and local ordering takes place irrespective of comonomer composition, although segmentization appears only in the case of low comonomer content copolymer. A molecular mechanism of chain extension has been proposed for the uniaxial elongational flow of COCs that fits well with the experimental data and can also be extended to any branched system or where there is an anchoring effect. Shear viscosity and subsequent glass-transition measurement also support the segmentization of lower comonomer content COCs. The rigidity of COCs increases with comonomer content, which makes it difficult to align toward a force field.

The author thanks Mitsui Chemical Inc. for providing COC samples with different compositions.

\section{References}

1. Kotaka, T.; Kojima, A.; Okamoto, M. Rheol Acta 1997, 36, 646.

2. Maiti, P.; Okamoto, M.; Kotaka, T. Polymer 2001, 42, 3939.

3. Maiti, P.; Okamoto, M.; Kotaka, T. Polymer 2001, 42, 9827.

4. Tokkyo-kouhou H4-48803 (Mitsui Petrochemical Inc.).

5. Meissner, J.; Hostettler, J. Rheol Acta 1994, 33, 1.

6. Haselwander, F. A.; Heitz, W.; Krugel, S. A.; Wendorff, J. H. Macromol Chem Phys 1996, 197, 3435

7. Rische, T.; Waddon, A. J.; Dickinson, L. C.; MacKnight, W. J. Macromolecules 1998, 31, 1871.

8. Chang, S.; Han, C. D. Macromolecules 1997, 302021.

9. Larson, R. G. The Structure and Rheology of Complex Fluids; Oxford University Press: New York, 1999.

10. Ugaz, V. M.; Burghardt, W. R. Macromolecules 1998, 31, 8474.

11. Ugaz, V. M.; Burghardt, W. R.; Zhou, W.; Kornfield, J. A. J Rheol 2001, 45, 1029.

12. Hongladarom, K.; Secakusuma, V.; Burghardt, W. R. J Rheol 1994, 38, 1505.

13. Soskey, P. R.; Witer, H. H. J Rheol 1984, 28, 625.

14. Doi, M; Edwards, S. F. The Theory of Polymer Dynamics; Oxford University Press: Oxford, UK, 1986.

15. Larson, R. G. J Rheol 1984, 28, 545.

16. Larson, R. G. J Rheol 1985, 29, 823. 